\title{
Physiotherapy-as-first-point-of-contact-service for patients with musculoskeletal complaints: understanding the challenges of implementation
}

\author{
Fiona Moffatt ${ }^{1}$, Rob Goodwin ${ }^{2}$ and Paul Hendrick ${ }^{1}$ \\ ${ }^{1}$ Assistant Professor, School of Health Sciences, University of Nottingham, Nottingham, UK \\ ${ }^{2}$ Doctoral Student, School of Medicine, University of Nottingham, Nottingham, UK
}

\begin{abstract}
Background: Primary care faces unprecedented challenges. A move towards a more comprehensive, multi-disciplinary service delivery model has been proposed as a means with which to secure more sustainable services for the future. One seemingly promising response has been the implementation of physiotherapy self-referral schemes, however there is a significant gap in the literature regarding implementation. Aim: This evaluation aimed to explore how the professionals and practice staff involved in the delivery of an in-practice physiotherapy self-referral scheme understood the service, with a focus on perceptions of value, barriers and impact. Design and setting: A qualitative evaluation was conducted across two UK city centre practices that had elected to participate in a pilot self-referral scheme offering 'physiotherapy-as-afirst-point-of-contact' for patients presenting with a musculoskeletal complaint. Methods: Individual and focus group interviews were conducted amongst participating physiotherapists, administration/reception staff, general practitioners (GPs) and one practice nurse (in their capacity as practice partner). Interview data were collected from a total of 14 individuals. Data were analysed using thematic analysis. Results: Three key themes were highlighted by this evaluation. First, the imperative of effecting a cultural change - including management of patient expectation with particular reference to the belief that GPs represented the 'legitimate choice', re-visioning contemporary primary care as a genuine team approach, and the physiotherapists' reconceptualisation of their role and practices. Second, the impact of the service on working practice across all stakeholders - specifically re-distribution of work to 'unburden' the GP, and the critical role of administration staff. Finally, beliefs regarding the nature and benefits of physiotherapeutic musculoskeletal expertise - fears regarding physiotherapists' ability to work autonomously or identify 'red flags' were unfounded. Conclusion: This qualitative evaluation draws on the themes to propose five key lessons which may be significant in predicting the success of implementing physiotherapy self-referral schemes.
\end{abstract}

Key words: implementation; musculoskeletal complaints; physiotherapy self-referral; primary care

Received 11 November 2016; revised 12 July 2017; accepted 17 August 2017;

first published online 12 September 2017

Correspondence to: Dr Fiona Moffatt, Assistant Professor, Division of Physiotherapy and Rehabilitation Sciences, School of Health Sciences, The University of Nottingham, B80 Clinical Sciences Building, City Hospital Campus, Nottingham, NG5 1PB, UK. Email: fiona.moffatt@nottingham.ac.uk

\section{Introduction}

It is widely accepted that the National Health Service faces an unprecedented productivity challenge (Ham, 2016). General practice, the 'cornerstone of the NHS' (p3) is no exception (National Health 
Service (NHS) England, 2014). It faces not only financial pressures, but also increasing public demand/expectations, issues with recruitment and retention, and the imperative to secure a sustainable service for the future (National Health Service (NHS) England, 2014). Within this challenge, healthcare professionals are identified as playing a critical role (Moffatt et al., 2014), in particular, identifying opportunities for greater innovation in clinical delivery (Appleby et al., 2014). One proposal is that primary care pursues more integrated models of provision, with specialist services provided by a comprehensive multidisciplinary team (NHS England, 2014).

One response to this has been the implementation of in-practice physiotherapy self-referral schemes where a physiotherapist, rather than the GP, is the first point of contact. This is a potentially significant solution to the challenges faced by primary care, given the suggested burden of musculoskeletal (MSK) problems - some authors proposing that these constitute the second leading reason for GP consultation (Foster et al., 2012). There is empirical evidence that physiotherapists are well placed to provide efficacious management of MSK complaints in general practice/primary care settings (Holdsworth and Webster, 2004; Ludvigsson and Enthoven, 2012; Goodwin and Hendrick, 2016) and that such approaches are associated with high levels of patientreported satisfaction (Webster et al., 2008; Goodwin and Hendrick, 2016). Nevertheless, only four in ten clinical commissioning groups in England offer Physiotherapy self-referral as a substantive or pilot service [Chartered Society of Physiotherapy (CSP), 2015a; 2015b]. Whilst patient, physiotherapist and GP perceptions of self-referral have been explored using mixed-methods surveys (Holdsworth et al., 2008; Webster et al., 2008), there is an apparent lacuna in empirical literature regarding the implementation of physiotherapy-as-first-point-ofcontact-service (PFPCS) for patients with MSK complaints. This paper describes a qualitative evaluation which aimed to address the gap by exploring how healthcare professionals and practice staff involved in the delivery of a PFPCS understood and perceived the service. It is proposed that the findings may inform and enable future implementation.

Specifically, the aims of this evaluation were to:

(1) Identify the value that stakeholders attribute to a PFPCS pilot service within primary care;
(2) Understand barriers to its effective implementation;

(3) Explore the impact of a PFPCS on the working practice of providers.

\section{Methods}

\section{Context}

A novel PFPCS was established and piloted in two GP practices (inner city practice and university campus practice) between April 2014 and March 2015. The practices were recruited as sites for the implementation of the PFPCS following an expression of interest invitation from the Clinical Commissioning Group.

Patients, presenting to either practice, were offered the opportunity to see an advanced practice physiotherapist (working at Agenda for Change band 7), as an alternative to usual GP care. These physiotherapists were able to assume full autonomy and responsibility for the patients' care. The intent was that the provision of advanced practice roles (eg, referral to secondary care, referral for diagnostic investigations), would optimise any potential unburdening of the GP. Nevertheless, physiotherapists were fully integrated within the primary care team and shared access to the electronic patient records which were visible to all providers.

Patients were given the choice to access the PFPCS following an explanation of the service by a member of the practice administration staff. ${ }^{1}$ If patients chose to see the physiotherapist, they were able to access up to two 20 minute appointments. The intent of this design was to replicate traditional GP care. Most patients were managed utilising a tailored self-management strategy. For those patients who had ongoing physiotherapy requirements, referrals were made to an appropriate primary care physiotherapy provider at the end of the second appointment. During the pilot scheme, two half day physiotherapy clinics per week were available at each practice. A total of 555 patients were seen by the PFPCS, with the majority requiring a single appointment only ( $78 \%$ inner city practice; $92 \%$ university campus practice). Almost all the patients were managed

\footnotetext{
${ }^{1}$ Administration staff were offered a training session prior to commencing the PFPCS, however not all individuals attended.
} 
independently, without referral to the GP (98\% inner city practice; $99 \%$ university campus practice). A proportion of patients were subsequently referred onwards for locally commissioned MSK physiotherapy services (11 patients, inner city practice; seven patients, university practice). Use of other resources is reported fully elsewhere (Goodwin and Hendrick, 2016). The safety of the PFPCS was analysed retrospectively by review of incidents reported by either the physiotherapists or general practices themselves. This was done through systematic subjective reporting and review of electronic incident reporting systems. No adverse incidents were recorded at either practice.

\section{Design}

A qualitative evaluation of the PFPCS (Murphy et al., 1998) was conducted after 12 months. Following Work Health Organization (WHO) guidelines, the scope was to learn from experiences, in order to 'improve current activities and promote better planning by careful selection of alternatives for future action' (WHO, 1981: 11). This qualitative evaluation was part of a mixedmethods approach [see Goodwin and Hendrick (2016) for details of the PFPCS quantitative evaluation and economic analysis]. Advice was sought from a local university research ethics committee, who confirmed that the project constituted a clinical service evaluation and therefore did not require formal ethical approval.

\section{Participants}

A purposive sampling process was applied recruiting GPs (and one nurse in their capacity as practice partner), physiotherapists and reception/ administrators from both pilot sites. A pragmatic approach to data collection was adopted, with interviews conducted individually where this was more convenient, or within small focus groups (see Table 1).

\section{Procedures}

Interviews took place in 2015 by a research assistant. All interviews were audio recorded with the duration of each interview ranging between 20 and $40 \mathrm{~min}$. All interviews took place in the respective practice. A semi-structured interview schedule was devised collaboratively by the research team, which permitted flexibility and
Table 1 Data collection methods and participants

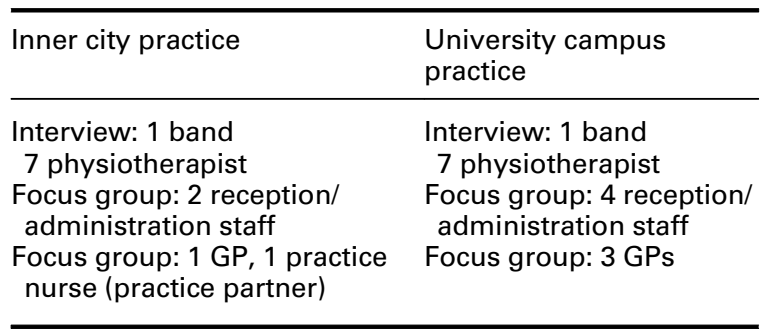

reflexivity. A nominated code (indicating their professional designation) was given to each participant in order to preserve confidentiality and anonymity.

\section{Analytic procedure}

Interview data were transcribed verbatim. Initial analysis was undertaken by one investigator (F.M.) and codes developed via an inductive thematic analysis (Braun and Clarke, 2006). The analytical framework was based upon improvement science theory, specifically the social determinants of action (Langley and Denis, 2011). The six stages of Braun and Clarke's (2006) guide to undertaking thematic analysis were adhered to, moving from the identification of initial codes to the generation of themes. The initial codes were subsequently checked and validated by R.G. and P.H. Any discrepancies were discussed by the whole group and consensus was reached. Themes were then generated by the research group as a whole.

\section{Results}

The findings of this qualitative evaluation were categorised according to three themes which were consistent across both practices: effecting a cultural change; working practice; and MSK expertise. Each theme addresses the service evaluation aims in elucidating the perceived value of, potential barriers to and impact of the service, as articulated by participants. These themes are presented in detail below.

\section{Effecting a cultural change}

All participants discussed the issue of managing patient expectations of a primary care service. Many referred to existing perceptions as 
problematic, specifically the belief that the GP represents the 'legitimate choice':

'I guess that there's still this culture that patients perceive the GP as being at the top of the hierarchy in terms of clinical experience... hopefully with the pilot that we've done here... they may start to recognise that, in actual fact, "if I've hurt my back or hurt my knee, I'd be much better going to see the physio..."

All professional groups acknowledged the imperative to address this preconception in order to ensure the success of the new service. The administration team however - essentially the first point of contact for the service - recognised that their (perceived) lack of professional authority made effecting this cultural change somewhat difficult:

'If a doctor says to them, "you'll be better off seeing the physio" they'll take the doctor's word for it, more than the receptionist's'

(Admin 1)

Both GPs and physiotherapists discussed the corollary effects of the PFPCS in terms of achieving such a cultural change, and reinforcing the wider message that contemporary primary care should be viewed as a team approach:

'It sort of increases the message that actually
primary care is a team offering to the citizen
rather than a GP offering... We've had the
nurse within primary care for a while... we
have health care assistants... this is another bit
of the offering. So... it builds on that cultural
change that is required across the system. You
know, it fits with that left shift out of hospital...
It's not GP, it is truly the primary care team'

(GP4)

The physiotherapists also acknowledged the need to reconceptualise the way in which they defined their professional role and working practices, however neither participants viewed this as problematic. A number of factors appeared to have supported this transition, namely support and interest from their professional body, access to full GP notes, adoption of a screening type assessment which was perceived to be more aligned to the way in which GPs worked, and a greater general understanding of inter-professional roles.

Primary Health Care Research \& Development 2018; 19: 121-130
This theme illustrates that the key cultural issues which appeared to be changing within these practices centred on reconceptualisation of inter-professional hierarchies of practice, and the changing perception of primary care services as being driven by a more multi-professional approach.

\section{Working practice}

All participants described the benefits of the PFPCS in terms of a strategy to help to manage the unprecedented demand faced by primary care. The justification for this was twofold: redistributing work and 'unburdening' the GP.

The administration staff and nurses highly valued the ability to redistribute work and have the ability to offer alternative appointments:

'We always need appointments... It was brilliant when you were doing telephone triage because you'd think, "Cor, I've got another appointment I can put them in"

(Nurse 1)

This ability to re-direct patients also had significant impact for the administration staff who frequently had to deal with patients frustrated by lack of capacity:

'It does help us... move the patient along quicker... and it takes the pressure off us...'

(Admin 1)

There was a widespread notion across all occupational groups that the service had 'unburdened' the GP. Whilst the GPs acknowledged this to an extent, the nature of the change was discussed in a very specific fashion. Whilst the national discourse had been one centred around physiotherapists reducing GP MSK workload by up to $30 \%$ (CSP, 2015a; 2015b), the GP participants in this study did not recognise such an impact. Their argument was that, invariably, the complexity of their patients meant that such a simplistic trade-off was not feasible:

'I think estimates range in the prevalence of musculoskeletal work in general practice... probably twenty, thirty percent. But... quite often that's one of a whole number of problems that they might bring to their GP. So it might be nice to think that seeing a physiotherapist might free the GP up for more 
time, but... quite often with the patient groups that we're seeing, they may be seeing the GP for other things, and it's an add on, it's an extra thing they want to squeeze in. So it may not have saved much time. It's a hard thing to quantify'

Uncertainty with regards 'quantification' of workload benefits for the GP was a frequently discussed confounder. The complexity of the GP workload was offered as justification for this:

'There's so many confounding factors around $G P$ workloads... it's really difficult to prove definitely that it's taken lots of work off. I'd say my feeling is, logically, common sense that's the case, but actually doing that in an absolutely rigorous way would be extremely hard... nailing it to an exact number's pretty difficult'

(GP4)

Although establishing metrics to demonstrate value for money was perceived to be difficult, GPs fully acknowledged the wider economic and emotional benefits of rapid access to a physiotherapy triage service, and a more 'joined up' service with other physiotherapy providers:

'If you have an injury and you have to wait 7 weeks [to access traditional out-patient physiotherapy services], that's lost the window of opportunity... the economic and emotional cost to that patient not being able to do stuff for several weeks until they get to the physio isn't great. Whereas, get in early, there's... a whole host of benefits'

Indeed, the benefits for patient experience and well-being were widely discussed and universally perceived as positive. As a primary care service, being able to offer a greater skill mix was viewed as empowering patients to make a decision about their preferred care, and therefore consistent with person-centred values. The physiotherapists discussed the overwhelmingly positive 'friends and family' data collected (NHS, 2016a), although acknowledged that this was predicated on appropriateness of referral.

All participants identified the critical role of the administration staff in terms of helping patients navigate their way into the service. Initially, this group of staff expressed wariness about the perceived responsibility, and anxiety with regards making errors:

'We was a bit, at first, unsure. We didn't want to make a mistake... because we was worried that we was booking them in with the physio when it could be something else... So in the beginning it was a bit hit and miss... and we weren't booking in as many as we should'

(Admin 1)

The data demonstrated that the PFPCS had influenced working practice, not solely in a strategic sense in terms of division of labour, but also in relation to providing a more person-centred, holistic service that was seen as particularly positive. Interestingly, any perceived benefits to GP workload were clouded by the complexity of the patient conditions presenting in primary care. Managing and ensuring the smooth running of the service was seen as an increased responsibility on the administrative team and thus had the potential to induce some degree of work-related stress, at least in the early stages of the pilot.

\section{MSK expertise}

The GP participants in this study all acknowledged that any concerns regarding the physiotherapists' ability to work autonomously and identify 'red flags' were unfounded. This view was also supported by the physiotherapists, who strongly defended their clinical reasoning as at least comparable with that of medically qualified practitioners:

'So I think that every patient has been appropriately managed and they've actually been screened very well. It could also work the other way because I saw two patients with missed fractures... One of them I sent for an $x$-ray because it was a quite longstanding thing. And that was picked up on the $x$-ray that I sent them for. The other one had been missed in Accident and Emergency, and I just sent them straight back... and that actually led to the $A$ and E consultant, sort of, putting in an informal complaint about the junior doctor who hadn't... x-rayed the ankle in line with $A$ and $E$ protocol... 
Maybe there's some things that we might be better at spotting'

Furthermore, the number of patients referred back to the GP, or the creation of 'on-work' was described as minimal. However, both GPs and physiotherapists believed that whilst the nature of the service 'expos[ed] physios to a certain degree of clinical risk' (PT1), its success was dependent upon having experienced MSK physiotherapists working in an extended scope role. Consequently they expressed concerns for the future of the service if this level of seniority was reduced:

'I worry a little bit if you were putting in more
junior, maybe cheaper, physiotherapists in,
that they might not be as effective... [the phy-
sio] has taken the patient and taken ownership
of them... and if it was someone less experi-
enced we might find that we were getting more
patients bounced back to us'

'This is an extended role... I think this needs to be a senior member of staff... and in an ideal world [one] who's got some sort of postgraduate training in advanced practice skills. Otherwise you are just trying to do it on the cheap, and maybe the quality of care might not be so good'

Whilst the GPs were highly satisfied with the service provided by the physiotherapists, there was a suggestion that some were still aware of the potential risk of something being missed. Indeed in one practice, the GPs spoke of the benefit of a shared information system. This offered a level of transparency that permitted the GP to 'check' that '[the patient's] history is taken, quite a thorough exam is performed and then there's a plan of action quite clearly documented' (GP3).

The high level of MSK expertise offered by the two physiotherapists was widely discussed amongst all the GPs. Indeed, some acknowledged that, 'actually, a lot of GPS do not have great MSK skills' (GP4), and that 'patients... are quite quick to pick up on that side of things and say... "I'd like to see a specialist". But actually, if they're seeing a physio who is very knowledgeable... it ticks that worry in their head' (GP4). This view was supported by the physiotherapists who both believed that, in general, they were able to help the patient navigate their therapeutic journey in a more efficient manner:

'My impression is that sometimes GPs may tend to use things like orthopaedics or diagnostics more, and the patient's not necessarily needing that and therefore that slows the patient journey, maybe makes it a bit more convoluted'

(PT1)

In addition to the value to the patients, this MSK expertise was seen as beneficial to the team. Both the GPs and the practice nurse discussed the opportunities for upskilling:

'I always read on [electronic notes] what they've done and what's happened, just to learn myself... I look at their assessments and their outcomes'

(Nurse 1)

'It's interesting to read [the physio's] thoughts on things. And that definitely up-skills us if we're reading about special tests that have been done'

(GP2)

One practice spoke of the imperative to better integrate the physiotherapists into the primary care team (as a pilot service, the physiotherapists had limited sessions within the practice), in order to both strengthen the two-way rapport and the two-way learning. However, whilst the PFPCS offered the potential for upskilling, there was also a pragmatic concern amongst some GPs that the 'siphoning off' of MSK cases would ultimately have a deleterious effect for their personal professional skills:

'This is a very effective service for patients, but it's also taking it away from the GPs... personally I like doing the musculoskeletal things, so I may be losing out a little bit'

This section shows how fears had been allayed to some degree, and health staff reassured by the knowledge and expertise of the physiotherapist, as well as the benefits that this brought to the patient, and the team in terms of learning and professional development. There was, however, acknowledgement that this service, with altered skill mix and expertise, could also been seen as 
potentially de-skilling GPs in the area of MSK practice.

\section{Discussion}

\section{Summary}

The new direction and model for primary care (NHS, 2016b) includes a vision of care provided by a multidisciplinary practice team that makes better use of the wider workforce, to promote quality, innovation, productivity and safety. This study examined one specific improvement initiative aligned to this aspiration: a PFPCS. The focus of this study was to explore implementation via the perspectives of certain stakeholders, specifically perceived value, barriers/facilitators and the impact on working practice. Key findings suggest that the PFPCS was perceived by all stakeholders in this study to add value. Principally, it was felt that patient experience could be enhanced by providing clinical expertise in MSK health, delivered by the physiotherapists. Furthermore, the upskilling and development of the wider general practice staff was acknowledged. The evaluation also identified some key barriers/facilitators, for example the importance of adequate training for all staff to ensure role clarity (perhaps most importantly for the administrative staff), and managing the socio-cultural expectations of patients. Finally, it was acknowledged that initiatives of this type could impact working practice and resource availability, although the metrics were perceived to be complex. The issue of clinical risk, whilst acknowledged, was believed to be mitigated by the use of expert physiotherapists. Certain perceptions were highlighted that need to be considered in future implementations; the perceptions that clear reductions in GP workload will be realised, and GPs' concerns regarding de-skilling.

\section{Comparison with existing literature}

The timeliness of this evaluation is demonstrated through the growing body of evidence considering the implementation of initiatives where physiotherapists provide the first point of contact for patients with MSK related health complaints (Foster et al., 2012; Ludvigsson and Enthoven, 2012; Goodwin and Hendrick, 2016; Bishop et al., 2017). The proliferation of such role development has been widely described in other occupations (Sibbald et al., 2004), not least of all in community and primary care nursing (Aranda and Jones, 2008; Hoare et al., 2012), and pharmacy (Dolovich et al., 2008).

There is also an extant literature which considers the implementation of service re-design as a tool to improve productivity within the general healthcare context (Appleby et al., 2014). Social science has made a significant contribution to this literature, with the premise that consideration of social structure and agency is imperative for those who wish to effect sustained improvements in practice, particularly in identifying barriers and enablers (Moffatt et al., 2016). The findings of this current evaluation are consistent with, and provide support for, this body of literature.

Dixon-Woods et al. (2012) analysed quality improvement evaluation reports to produce generalisable insights into likely challenges, and suggestions of ways in which future improvement projects could be ameliorated. The findings of this study are consistent with a number of these. For example, these authors also highlighted the imperative to consider organisational and professional culture, recommending that multiple stakeholders: understood the demands and roles via access to relevant information; had adequate support and training; and could identify the boundaries of responsibility and accountability. In addition, they supported the use of a relevant programme theory (The Health Foundation, 2011) whereby clearly established and meaningful outcomes could be causally linked to the interventions undertaken. In the evaluation of the PFPCS, the difficulty in accurately assessing the impact of the service on GP workload was indeed an acknowledged problem. Finally, Dixon-Woods et al. (2012) noted the challenge of unintended consequences. This was similarly noted within the PFPCS evaluation, particularly the potential of the service to de-skill the GPs.

What this study adds, is the imperative to consider the wider socio-cultural issues for PFPCS initiatives in terms of patient perception of primary care as a hierarchical structure. Elliott et al. (2011) noted that, in their study of 8000 adults across 20 UK general practices, patients visited 'other' primary healthcare professionals (as an alternative to the GP) in less than $2 \%$ of symptom episodes. 
These authors do not pass comment on the explanation for such low uptake of alternative primary care services, but rather suggest that further research is justified. It has previously been suggested that societal values (particularly amongst older patients) influence the belief that the doctor is the only appropriate person to make a diagnosis (Wilson et al., 2002).

\section{Implications for practice}

Lesson 1: A change in 'culture' is essential for all if the service is to be deemed a rational choice by patients

Participants in this study highlighted that a potential barrier to the implementation of a PFPCS could be the patient perception of primary care as a hierarchical structure, with the GP as the only 'rational choice' for diagnosis and management of a MSK complaint. It is not unreasonable to hypothesise that a possible solution might be a richer exchange of information, in order to alter perceptions, empower patients to make an informed choice, and create a shared understanding. A PFPCS should be actively promoted and signposted by GPs and other team members (Rimmer, 2011), and information and education offered regarding the nature of physiotherapy expertise (Wilson et al., 2002; Holdsworth and Webster, 2006).

Lesson 2: Practices must be cognisant of the critical role played by administration staff

The findings of this qualitative evaluation demonstrate that the PFPCS resulted in a range of perceived challenges and benefits. The service was viewed extremely positively for its ability to create capacity within the general practice system and thereby reduce stress, particularly for frontline administration staff who were primarily in charge of the booking systems. Emotional labour and stress is endemic within general practice (Lown et al., 2015) and is not restricted to clinical staff (Ward and McMurray, 2011; Murray, 2014). Our results indicate that the administration team should be acknowledged as integral to managing the process/structure and delivery of the PFPCS service. The provision of formal training for these staff members should be considered in order that they can filter patients appropriately and confidently.

Primary Health Care Research \& Development 2018; 19: 121-130
Lesson 3: If services are built and promoted purely on the premise that they will reduce GP workload by $30 \%$, they will arguably fail to meet that target

It has been hypothesised that, given the perceived ability of PFPCS to create capacity, the alleged benefit of 'unburdening' the GP would be realised (Greenfield et al., 2016; NHS, 2016b). However, in these practices, any direct benefits to GP workload were, in some part, affected by the complexity of the presenting patients. Data suggests that one in six UK patients has more than one of the conditions listed within the Quality and Outcomes Framework, and that this cohort of complex patients accounts for around one-third of GP consultations (Salisbury et al., 2011). These findings are in keeping with the views expressed by the GP participants in this evaluation, that is that patients commonly present with MSK problems, but as co-morbidities to another condition. As such, the GPs perceived that their workload had not necessarily decreased, or at least not to the extent that had potentially been anticipated. Contemporary literature acknowledges that evaluation of schemes involving relaxation of GP gatekeeping are essential, including the impact on use of care and workload (Greenfield et al., 2016). Future studies should also consider alternative metrics, including patient experience/ satisfaction and clinical outcomes.

Lesson 4: PFPCS provides an opportunity to develop expertise in MSK across the team - but must be mindful of potential for de-skilling the GP

An additional benefit perceived by clinical staff in this evaluation was the widening of the skill mix within the practices. The benefits and challenges of an increased skill mix within primary care have long been recognised (Rashid et al., 1996). In the context of this evaluation, the enhanced skill mix was viewed as critical for effecting a cultural change for the service users, and also the opportunity for 'upskilling' of resident clinicians, either via formal or informal educational opportunities from the physiotherapist. There were however perceived problems, specifically the view of certain GPs that the service had the potential to 'de-skill' them in MSK diagnosis and management.

Williams and Sibbald argue that 'changing roles and identities across professional boundaries create a culture of uncertainty, a process which paradoxically has the potential both to inspire innovation and to threaten innovation in primary health 
care' (1999: 737). We recommend that for successful integration of a PFPCS, service design should pay attention to incorporating formal opportunities for multi-professional team training, shared tools (eg, IT systems and records, assessment proformas, etc.) and case discussion sessions.

\section{Lesson 5: Issues of responsibility and accountability can be addressed}

A significant anxiety regarding implementation of physiotherapy self-referral schemes has concerned issues of responsibility and accountability, particularly the worry that patient safety could be compromised if non-musculoskeletal issues were overlooked (Bley Griffiths, 2015). In this evaluation however, the participants' fears around safety had been allayed. A caveat to this was the belief that a PFPCS should be delivered by a clinician working at an advanced level and with specialist training. To aid implementation of PFPCS, GPs may benefit from information regarding the training undertaken by physiotherapists and their professional registration requirements, as well as personal experience of working with a physiotherapist to engender confidence (Wilson et al., 2002; Holdsworth and Webster, 2006).

\section{Strengths, limitations and further research}

This study was novel in its intent to qualitatively explore the challenges of implementing physiotherapy self-referral within general practice, as perceived by some of the key stakeholders within the service. It combined empirical data with social science theory to gain relevant understandings. It is possible that alternative insights may have been elucidated had a larger and more diverse sample been used; participants were drawn from only two general practices and as such the results are context specific. Patients' perspectives were not sought in this qualitative evaluation; an evaluation of the patient perspective is ongoing. Given the key theme concerning socio-cultural expectations, future research should consider elucidating this perspective. Furthermore, this evaluation took place just over 12 months from the PFPCS being initiated. Cultural changes in practice delivery and stakeholder perceptions might be expected to take years to become evident. Additional data collection methods may also be warranted for future work, for example document/artefact analysis and observation. Further quantitative evaluation would also be illuminating particularly with regards to the perceived hierarchy in primary care; data regarding the number of patients who declined PFPCS in favour of the GP would be of significance. Finally, whilst GPs' concerns regarding the autonomous work by physiotherapists were unfounded in this study, further large-scale, longitudinal studies should explore patient safety.

\section{Acknowledgements}

The authors thank Dr Martin Willis, the research assistant who completed the interviews. The authors also thank the practice staff who contributed to this study.

\section{Financial Support}

This research received no specific grant from any funding agency, commercial or not-for-profit sectors.

\section{Conflicts of Interest}

None.

\section{References}

Appleby, J., Galea, A. and Murray, R. 2014: The NHS productivity challenge: experience from the front line. London: The King's Fund.

Aranda, K. and Jones, A. 2008: Exploring new advanced practice roles in community nursing: a critique. Nursing Inquiry 15, 3-10.

Bishop, A., Ogollah, R.O., Jowett, S., Kigozi, J., Tooth, S., Protheroe, J., Hay, E.M., Salisbury, C., Foster, N.E. and The STEMS Study Team 2017: STEMS pilot trial: a pilot cluster randomised controlled trial to investigate the addition of patient direct access to physiotherapy to usual GP-led primary care for adults with musculoskeletal pain. BMJ Open 7, e012987.

Bley Griffiths, E. 2015: Physiotherapists' call for patient selfreferral shot down by RCGP (WWW Document). Pulse Today. Retrieved 20 June 2016 from http://www.pulsetoday. co.uk/news/clinical-news/physiotherapists-call-for-patient-selfreferral-shot-down-by-rcgp/20009792.fullarticle.

Braun, V. and Clarke, V. 2006: Using thematic analysis in psychology. Qualitative Research in Psychology 3, 77-101.

Chartered Society of Physiotherapy 2015a: Physiotherapy works: self-referral (WWW document). Retrieved 9 August 2016 from http://www.csp.org.uk/professional-union/ practice/your-business/evidence-base/physiotherapy-works/selfreferral. 
Chartered Society of Physiotherapy 2015b: Place physiotherapists on the frontline to free up millions of GP appointments, says CSP (WWW document). Retrieved 16 June 2016 from http://www.csp.org.uk/press-releases/2015/04/22/placephysiotherapists-frontline-free-millions-gp-appointments-says-csp.

Dixon-Woods, M., McNicol, S. and Martin, G. 2012: Ten challenges in improving quality in healthcare: lessons from the Health Foundation's programme evaluations and relevant literature. BMJ Quality \& Safety 21, 576.

Dolovich, L., Pottie, K., Kaczorowski, J., Farrell, B., Austin, Z., Rodriguez, C., Gaebel, K. and Sellors, C. 2008: Integrating family medicine and pharmacy to advance primary care therapeutics. Clinical Pharmacology and Therapeutics. 83, 913-17.

Elliott, A.M., McAteer, A. and Hannaford, P.C. 2011: Revisiting the symptom iceberg in today's primary care: results from a UK population survey. BMC Family Practice 12, 16.

Foster, N.E., Hartvigsen, J. and Croft, P.R. 2012: Taking responsibility for the early assessment and treatment of patients with musculoskeletal pain: a review and critical analysis. Arthritis Research \& Therapy 14, 1-9.

Goodwin, R.W. and Hendrick, P.A. 2016: Physiotherapy as a first point of contact in general practice: a solution to a growing problem? Prim. Health Care Research \& Development 17, 489-502.

Greenfield, G., Foley, K. and Majeed, A. 2016: Rethinking primary care's gatekeeper role. BMJ 354, i4803.

Ham, C. 2016: The NHS must focus on better value in 2016 to deliver the $£ 22$ billion productivity challenge (WWW document). Kings Fund. Retrieved 14 January 2016 from http://www. kingsfund.org.uk/blog/2016/01/nhs-focus-better-value-2016.

Hoare, K.J., Mills, J. and Francis, K. 2012: The role of Government policy in supporting nurse-led care in general practice in the United Kingdom, New Zealand and Australia: an adapted realist review. Journal of Advanced Nursing 68, 963-80.

Holdsworth, L. and Webster, V. 2006. Patient self referral: a guide for therapists, 1st edition. Abingdon: Radcliffe Publishing.

Holdsworth, L.K. and Webster, V.S. 2004: Direct access to physiotherapy in primary care: now? - and into the future? Physiotherapy 90, 64-72.

Holdsworth, L.K., Webster, V.S. and McFadyen, A.K. 2008: Physiotherapists' and general practitioners' views of selfreferral and physiotherapy scope of practice: results from a national trial. Physiotherapy 94, 236-43.

Langley, A. and Denis, J.-L. 2011: Beyond evidence: the micropolitics of improvement. BMJ Quality \& Safety 20, i43-46.

Lown, M., Lewith, G., Simon, C. and Peters, D. 2015: Resilience: what is it, why do we need it, and can it help us? British Journal of General Practice 65, e708-710.

Ludvigsson, M.L. and Enthoven, P. 2012: Evaluation of physiotherapists as primary assessors of patients with musculoskeletal disorders seeking primary health care. Physiotherapy 98, 131-37.

Moffatt, F., Martin, P. and Timmons, S. 2014: Constructing notions of healthcare productivity: the call for a new professionalism? Sociology of Health \& Illness 36, 686-702.

Primary Health Care Research \& Development 2018; 19: 121-130
Moffatt, F., Timmons, S. and Coffey, F. 2016: ED healthcare professionals and their notions of productivity. Emergency Medicine Journal 33, 789-93.

Murphy, E., Dingwall, R., Greatbatch, D., Parker, S. and Watson, P. 1998: Qualitative research methods in health technology assessment: a review of the literature. Health Technology Assessment (Winchester, England) 2, iii-x, $1-274$.

Murray, H. 2014: Stress levels for practice managers at an all time high (WWW document). Retrieved 20 June 2016 from http://www.firstpracticemanagement.co.uk/blog/posts/practicemanager-stress-level-at-an-all-time-high/.

National Health Service (NHS) 2016a: NHS friends and family test - patient feedback - NHS choices (WWW document). Retrieved 16 June 2016 from http://www.nhs.uk/NHSEngland/ AboutNHSservices/Pages/nhs-friends-and-family-test.aspx.

National Health Service (NHS) 2016b. General practice forward view. London: NHS.

National Health Service (NHS) England 2014: Improving general practice: a call to action (phase 1 report).

Rashid, A., Watts, A., Lenehan, C. and Haslam, D. 1996: Skillmix in primary care: sharing clinical workload and understanding professional roles. British Journal of General Practice 46, 639-40.

Rimmer, A. 2011: Patients stick to GP services, despite alternatives, GP online (WWW document). Retrieved 20 June 2016 from http://www.gponline.com/patients-stick-gpservices-despite-alternatives/article/1065714.

Salisbury, C., Johnson, L., Purdy, S., Valderas, J.M. and Montgomery, A.A. 2011: Epidemiology and impact of multimorbidity in primary care: a retrospective cohort study. British Journal of General Practice: The Journal of the Royal College of General Practitioners 61, e12-21.

Sibbald, B., Shen, J. and Mcbride, A. 2004: Changing the skillmix of the health care workforce. Journal of Health Service Research \& Policy 9, 28-38.

The Health Foundation 2011: Learning report: safer patients initiative (WWW document). Retrieved 12 August 2016 from http://www.health.org.uk/publication/learning-reportsafer-patients-initiative.

Ward, J. and McMurray, R. 2011: The unspoken work of general practitioner receptionists: a re-examination of emotion management in primary care. Social Science \& Medicine 72, 1583-587.

Webster, V.S., Holdsworth, L.K., McFadyen, A.K. and Little, H. 2008: Self-referral, access and physiotherapy: patients' knowledge and attitudes - results of a national trial. Physiotherapy 94, 141-49.

Williams, A. and Sibbald, B. 1999: Changing roles and identities in primary health care: exploring a culture of uncertainty. Journal of Advance Nursing 29, 737-45.

Wilson, A., Pearson, D. and Hassey, A. 2002: Barriers to developing the nurse practitioner role in primary care - the GP perspective. Family Practice 19, 641-46.

World Health Organization 1981. Health programme evaluation. Geneva: WHO. 